\title{
BORDER IDENTIFICATION OF ECHOCARDIOGRAMS VIA MULTISCALE EDGE DETECTION AND SHAPE MODELING
}

\author{
Andrew Laine and Xuli Zong \\ Department of Computer \& Information Science \& Engineering \\ University of Florida, CSE Room 301, P.O. Box 116120, Gainesville, FL 32611 \\ E-mail: laine@cise.ufl.edu, zxl@cise.ufl.edu
}

\begin{abstract}
An algorithm for endocardial and epicardial border identification of the left ventricle in 2-D short-axis echocardiographic images is presented. Our approach relies on shape modeling of endocardial and epicardial boundaries and prominent border information extracted from image sequences. The algorithm consists of four steps; waveletbased edge detection, border segment extraction, border reconstruction, and boundary smoothing. Wavelet maximum representation of edges, dynamic shape modeling and matched filtering techniques are utilized to determine the center point of the left ventricle, and carry out feature extraction of border segments to better approximate endocardial and epicardial boundaries. The algorithm can reliably estimate the center point of the left ventricle, and also determine both endocardial and epicardial boundaries. Myocardial boundary identification is autonomous requiring no human input for initial estimation of boundary locations. Sample experimental results are shown for endocardial and epicardial border identification in 2-D short-axis echocardiograms.
\end{abstract}

\section{INTRODUCTION}

Automatic identification of endocardial and epicardial boundaries of the left ventricle (LV) has been a focus of attention for many researchers employing computational methods to assist cardiologists in screening clinical heart disease and diagnosis. Several approaches for boundary detection of the LV have shown partial success in either identifying the endocardial boundary $[1,2,3,4]$, the center point of the LV and partial boundary points $[5,6]$, or epicardial boundary detection [7]. In these (and most other) approaches, human knowledge of the approximate shape of endocardial and epicardial boundaries provided by a technician and/or a computer generated center point of the LV have been used successfully for boundary detection. Thus, modeling and analysis of the LV shape [8] have played important roles in previous boundary detection methods.

In our approach [9], four major steps are used to carry out the detection of endocardial and epicardial boundaries. First, a dyadic wavelet transform (DWT) [10, 11, 12, 13] is used to locate edge information for both boundary segments and other sharp intensity variation points. Secondly we use dynamic shape modeling and matched template filtering to estimate the center point of the left ventricle and extract candidate borders for each deformable endocardial and epicardial boundary. In the short-axis view of the LV, endocardial and epicardial boundaries are modeled as deformable elliptical shapes. Since the quality of ultrasound images is generally poor due to signal dropout and speckle noise the complete contours of endocardial and epicardial boundaries are difficult to obtain. First, we link candidate border segments to reconstruct closed contours. Finally, the linked closed contours are smoothed through local nonlinear filtering [14] without shrinkage, to obtain a reliable estimation of the true endocardial and epicardial boundaries.

This paper is organized as follows. In Section 2, we describe details of the methods for border identification of the LV. This includes a DWT for edge detection and shape modeling of the LV. The complete algorithm for identifying the center point of the LV and determining endocardial and epicardial boundaries of the LV is then presented. Sample experimental results are shown in Section 3. Finally, Section 4 presents our conclusions and summary.

\section{METHODOLOGY AND ALGORITHM FOR BOUNDARY DETECTION}

The methods behind the boundary detection algorithm are quite natural and straightforward [9]. With current technology, available ultrasonic images are often embedded with a considerable amount of speckle noise and signal dropout. The consequence is that it is difficult to find closed contours of endocardial and epicardial boundaries of the LV which are well delineated within an image sequence. "A priori" knowledge about the general shape of the LV has been successfully used in boundary detection algorithms. In our implementation, we combine high-level shape descriptors and primitive boundary features to reconstruct closed boundary estimates. Four steps are needed to carry out detection of endocardial and epicardial boundaries of the LV;

(1) Multiscale wavelet analysis for edge detection,

(2) Matched filtering for feature extraction,

(3) Boundary contour reconstruction, and

(4) Smoothing the boundary contours.

In our method of boundary detection, we first use a DWT to find edge information for each ultrasound image through local maxima [10]. The shape of the LV is then defined by a dynamically adjustable model $[5,6]$. Using shape 
modeling, we then carry out shape matched filtering to determine the center point of the LV and extract important boundary segments. The extracted boundary segments are then connected and smoothed to obtain an approximation of endocardial and epicardial boundaries of the LV.

\subsection{Wavelet-Based Edge Detection}

Local maxima representations of a DWT [10, 11, 12] are first applied for multiscale edge detection. An advantage provided by this wavelet-based edge detection method for echocardiograms is that the edge information at some middle level of frequency-space provides an accurate location of heart boundaries with less noise for more reliable feature extraction. Since we are interested in large structures, such as endocardial and epicardial boundaries, we discard the fine detail edges and focus only on edge maps within the middle levels of scale. The level of scale identified depends on selected basis wavelets adopted for a DWT and structure size relative to the support of each wavelet. For a basis wavelet, edge maps at level 3 provided more coherent structural information on our test echocardiographic images. However, for another basis, edges at level 4 demonstrated a better compromise. A small threshold 4.0 was applied to discard any edge points induced by speckle or background noise.

\subsection{Matched Filtering for Feature Extraction}

As part of the operations within this step, a pair of circular arc templates were generated and shape-matched filtering was performed to estimate the center point of the LV using the edge map obtained from Step 1. This was accomplished by first using an average-sized circular arc template to find the posterior epicardial boundary segments and determine the potential center point of the lower half of the LV. Next, an upper circular arc template with the same size as the lower circular arc template was generated and used to find the anterior epicardial boundary segments and to determine the potential center point of the upper half of the LV. The second matched filtering was carried out near the lower potential center point since the posterior epicardial border is one of the most reliable features found on 2-D short-axis echocardiograms $[5,6]$. The estimated center point of the LV was identified as the center point of the line segment linking the two potential center points.

After estimating the center point and rough locations of anterior and posterior epicardial borders, we then estimated wall thickness and constructed two concentric elliptic-shaped band masks for extracting both endocardial and epicardial boundary segments. This was accomplished via a simple masking operation. The band width of each mask specified how much deformation of myocardium wall was allowed during feature extraction. Postprocessing after masking allowed us to remove extracted noise edge points which may include weak boundary segments. Thus, length thresholding was applied on all extracted segments. The removal of weak boundary segments generally did not affect boundary reconstruction as long as enough boundary segments remained.

\subsection{Boundary Contour Reconstruction}

Next, boundary segments obtained from the previous steps were connected to reconstruct closed boundary contours. First, a preprocessing procedure was performed to connect broken boundary segments belonging to large boundary segments via an 8-neighbor connection and excessive points attached to a boundary segment were removed. An attached point was an edge point whose removal would not break the boundary segment to which it was attached.

During boundary contour reconstruction, we avoided mislinking two neighbor boundary segments by examining their relative locations, orientations, and distances. To avoid linking an endocardial boundary segment to an epicardial boundary segment, endocardial border segments were labeled distinctly from epicardial border segments during feature extraction in the previous step. We used a circular arc relative to the center point of the LV instead of a straight line to interpolate between the corresponding end points of each two-neighbor boundary segment. If the link procedure successfully connected all boundary segments, we obtained closed endocardial and epicardial boundary contours. If it failed to connect boundary segments because of their positions relative to each other or insufficient boundary segments, then only partial boundary points were realized. If a wrong connection (along the expected boundary contour) occurred, error between the detected boundary and the expected boundary was large, and we observed that the detected contour was distorted slightly relative to the predicted boundary contour.

\subsection{Smoothing of a Closed Contour Without Shrinkage}

For a better approximation of the endocardial and epicardial boundaries of the $L V$, we needed to smooth the reconstructed boundary contours. Two local smoothing filters were investigated. One was a window spatial-averaging smoothness filter [15] and the other was a non-shrinking local reproducible smoothness filter [14]. The first carried out traditional spatial averaging with distinct window sizes, and was satisfactory for subtle smoothing. However, when more smoothing was required, it shrunk the contour slightly. The second method applied was local reproducible smoothing without shrinkage [14]. Both smoothing algorithms were implemented for comparative purposes.

The non-shrinking local smoothness filter was selected for smoothing the endocardial and epicardial boundaries of the LV. As pointed out by Oliensis [14], this local smoothness filter has several advantages over other methods such as Gaussian filtering: (1) It is local, (2) It introduces no shrinkage of curves, (3) The filter can be easily implemented and smoothness of contours can be controlled by a single parameter. This local smoothness filter is essentially behaved as a non-linear low-pass filter.

\section{EXPERIMENTAL RESULTS}

The test images used in this study included several sequences of 2-D short-axis echocardiographic images of the 


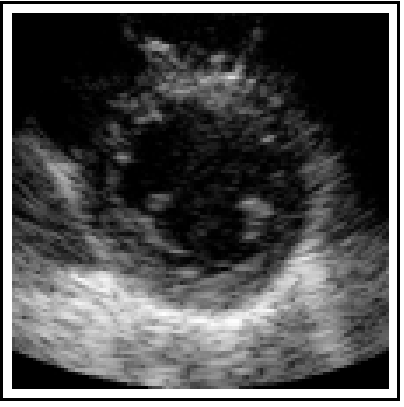

(a)

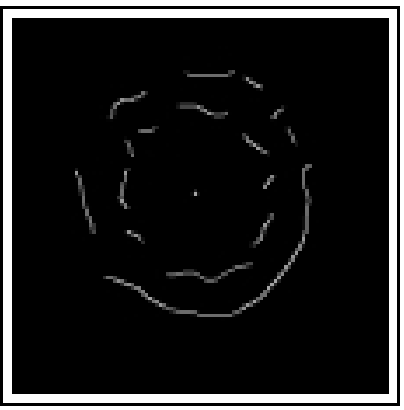

(c)

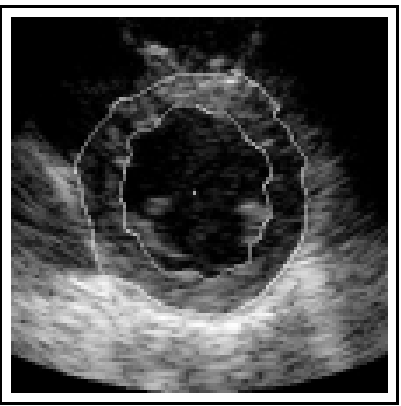

(e)

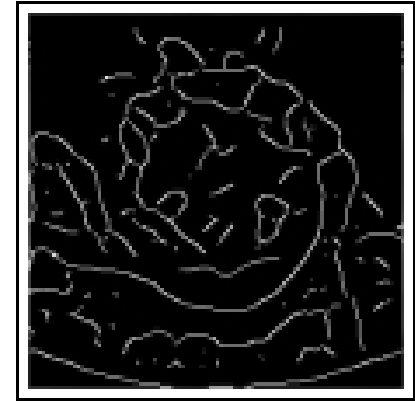

(b)

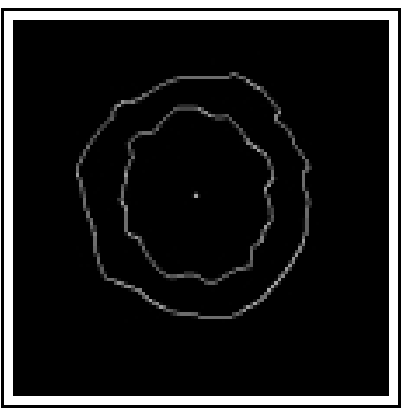

(d)

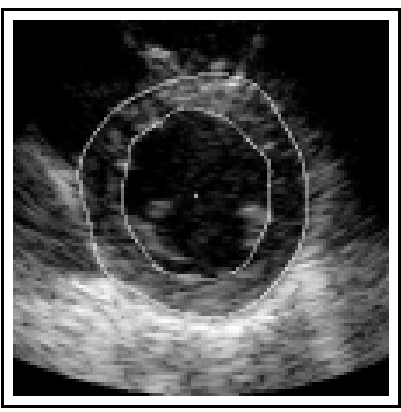

(f)

Figure 1. Border identification of the LV from a short-axis view. (a). An original frame of the LV, (b). Edge maps detected using a DWT, (c). The center point of the LV and extracted boundary segments, (d). Connected boundary contours, (e). Contours in (d) overlayed with the original, and (f). Final estimated boundaries.

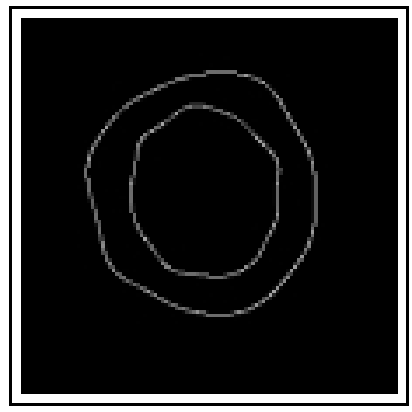

(a)

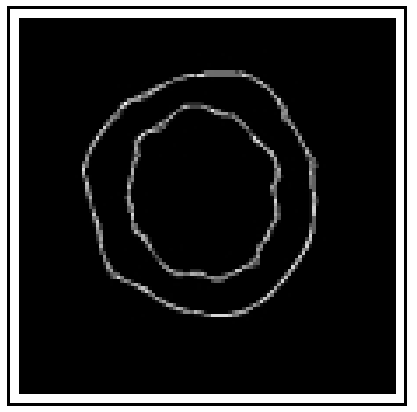

(b)

Figure 2. Local non-shrinking smoothness filtering of a closed contour. (a). The smoothed contours and (b). Contours in (a) overlayed with the contours in Figure 1(d) before smoothness filtering.

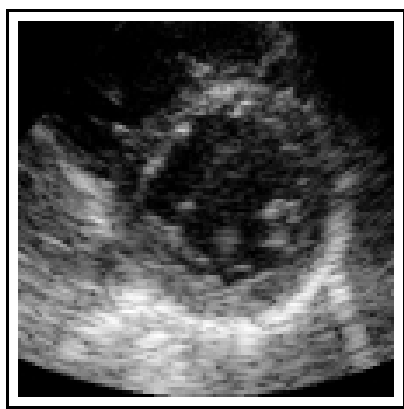

(a)

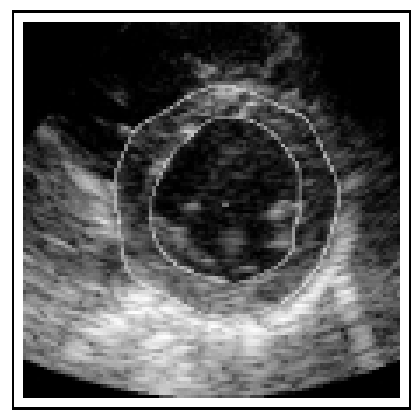

(b)
Figure 3. Border identification of a echocardiogram at ED (a). An original frame of the LV at ED and (b). The detected center point and endocardial as well as epicardial boundaries overlayed with the original.

LV. ${ }^{1}$ Each image was originally in polar format with a matrix size of $128 \times 512$ and 256 gray levels. These images were first converted to Cartesian coordinates and a size of 256x256 was cropped containing the entire LV for boundary detection.

Figure 1. shows sample results from the four major steps of our algorithm for endocardial and epicardial boundary detection of the LV in a 2-D short-axis echocardiogram. The original frame of the LV is given in Figure 1(a) which is near end diastole. Edge maps detected using a DWT at spatial-frequency level 3 are shown in Figure 1(b). The result of feature extraction for determining the center point of the LV as well as extracting boundary segments with noisy edge points removed is presented in Figure 1(c). Figure $1(\mathrm{~d})$ shows two closed contours after boundary contour reconstruction. The closed contours in Figure 1(d) are overlayed with the original image and presented in Figure 1(e) to show that the reconstructed endocardial and epicardial boundaries of the LV fit the image well, although the shape of the boundary contours appear less natural compared to real heart boundaries. The final smoothed boundary contours overlayed with the original image are shown in Figure 1 (f). The advantage of non-shrinking through local smoothing of a closed contour is made obvious in Figure 2. Distinct smoothing effects can be achieved by simply adjusting the smoothness factor. Another sample result of boundary detection on a frame from a different sequence of echocardiograms at end diastole (ED) is shown in Figure 3. Figure 4 provides the result image of the boundary detection for a frame at end systole (ES) from the same sequence as Figure 3 . These two boundary detection results are overlayed with their corresponding original images to demonstrate typical algorithm performance.

\section{CONCLUSIONS}

In this paper we have described a new algorithm for border identification of the LV in 2-D short-axis echocardiographic images. Four major steps were used to accomplish bound-

\footnotetext{
${ }^{1}$ These images were provided by Echocardiography Research Laboratory, Department of Medicine, University of Florida, Gainesville.
} 


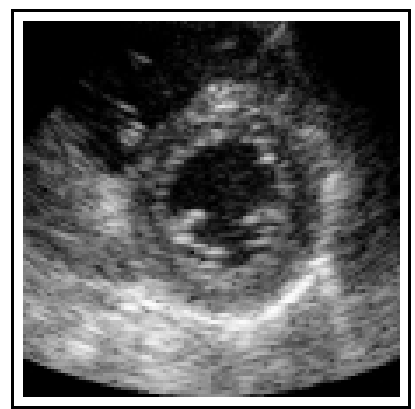

(a)

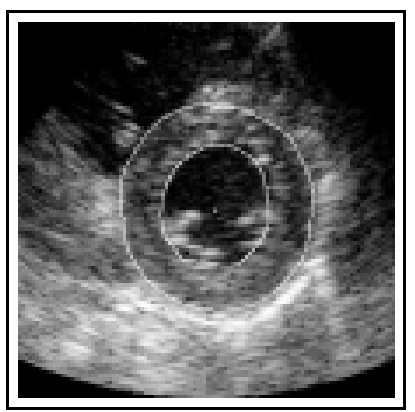

(b)
Figure 4. Border identification of a frame at ES from the same sequence of echocardiograms as Figure 3 (a). An original frame of the LV at ES and (b). The detected center point and boundaries overlayed with the original.

ary detection in a simple and straightfor ward manner. Multiscale wavelet-based edge detection was employed to locate sharp variation points at the local maxima of a DWT which included myocardial boundary points. Matched filtering and masking operations were then used for feature detection and extraction, including the center point of the LV and candidates of myocardial boundaries. Reconstruction provided closed piece-wise smoothed contours of endocardial and epicardial boundaries of the LV. Smoothing without shrinkage of closed contours provided a better approximation of expected endocardial and epicardial boundaries. Experimental results showed that the detected center point of the LV and two myocardial boundary contours were close to human expectation.

Similar to previously proposed methods $[2,5,6]$, this algorithm not only estimates the center point of the $\mathrm{LV}$, but also determines both endocardial and epicardial boundaries. Preliminary results suggest that this algorithm can provide reliable measurements for quantification. However, further improvements are needed, such as to further reduce the interference of undesired edge segments and noise. Additional optimization of this algorithm and measuring boundary detection performance on more complex and poorer quality images will be the focus of future research. The current algorithm may fail to produce closed myocardial boundary contours if few boundary segments are extracted. During boundary contour reconstruction, combining extracted boundary segments with estimated boundary segments based on shape modeling may help to overcome this problem.

Acknowledgments: the authors wish to thank Dr. Edward Geiser and Dr. David Wilson of the Departments of Medicine and Mathematics respectively, University of Florida, Gainesville, for their support and valuable suggestions.

\section{REFERENCES}

[1] L. Cohen, "On active contour models and balloons," CVGIP: Image Understanding, vol. 53, no. 2, pp. 211218, March, 1991.

[2] P. Detmer, G. Bashein, and R. Martin, "Matched filter identification of left-ventricular endocardial borders in transesophageal echocardiograms," IEEE Trans. Medical Imaging, vol. 9, no. 4, pp. 396-404, Dec., 1990.

[3] N. Friedland and D. Adam, "Automatic ventricular cavity boundary detection from sequential ultrasound images using simulated annealing," IEEE Trans. Medical Imaging, vol. 8, no. 4. pp. 344-353, Dec. 1989.

[4] S. Tamura, et al, "Three-dimensional reconstruction of echocardiograms based on orthogonal sections," Pattern Recognition, vol. 18, no. 2, pp. 115-124, 1985.

[5] D. Wilson and E. Geiser, "Automatic center point determination in two-dimensional short-axis echocardiographic images," Pattern Recognition, vol. 25, no. 9, pp. 893-900, 1992.

[6] D. Wilson, E. Geiser, and J. Li, "Feature extraction in two-dimensional short-axis echocardiographic images," J. of Mathematical Imaging and Vision, no. 3, pp. 285298, 1993.

[7] J. Feng, W. Lin, and C. Chen, "Epicardial boundary detection using fuzzy reasoning," IEEE Trans. Medical Imaging, vol. 10, no. 2, pp. 187-199, June 1991.

[8] C. Chen, T. Huang, and M. Arrott, "Modeling, analysis, and visualization of left ventrical shape and motion by hierarchical decomposition," IEEE Trans. Pattern Anal. Mach. Intell., vol. 16, no. 4, pp. 342-356, April 1994.

[9] A. Laine and X. Zong, "Quantification of echocardiograms by multiscale wavelet analysis and shape modeling," CISE Technical Report, Department of Computer \& Information Science \& Engineering, University of Florida, Gainesville, FL, 1996.

[10] S. Mallat and S. Zhong, "Characterization of signals from multiscale edges," IEEE Trans. Pattern Anal. Mach. Intell., vol. 14, no. 7, pp. 710, 1992

[11] A. Laine and S. Song, "Multiscale wavelet representations for mammographic feature analysis," Proc. of SPIE Conf. on Math. Methods in Medical Imaging, San Diego, CA, July 23-25, 1992.

[12] X. Zong, E. Geiser, A. Laine, and D. Wilson, "Homomorphic wavelet shrinkage and feature emphasis for speckle reduction and enhancement of echocardiographic images," Proceedings of SPIE's Medical Imaging 96: Image Processing, vol. 2710, pp. 658-667, Newport Beach, CA, February 12-15, 1996.

[13] X. Zong, A. Laine, E. Geiser, and D. Wilson, "Denoising and contrast enhancement via wavelet shrinkage and nonlinear adaptive gain," Wavelet Applications III, Proceedings of SPIE, vol. 2762, pp. 566-574, Orlando, FL, April 8-12, 1996.

[14] J. Oliensis, "Local reproducible smoothing without shrinkage," Proceeding (IEEE Computer Society Conference on Computer Vision \& Pattern Recognition), pp. 277-282, 1992.

[15] A. Jain, Fundamentals of digital image processing, Prentice-Hall, Inc., Englewood Cliffs, New Jersey, 1989. 